\title{
Metformin use in pregnancy: promises and uncertainties
}

\author{
Robert S. Lindsay ${ }^{1}$ - Mary R. Loeken ${ }^{2}$
}

Received: 27 March 2017 / Accepted: 22 May 2017 /Published online: 2 August 2017

(C) The Author(s) 2017. This article is an open access publication

\begin{abstract}
Metformin has been prescribed in pregnancy for over 40 years; for much of this time, use has been limited both in numbers and geographically, and the evidence base has been confined to observational studies. In early years, perceived safety concerns and lack of availability of the drug in many countries acted as a barrier to use. More recently, RCTs have begun to examine the role of metformin in pregnancy in much-needed detail. However, this evidence base has been interpreted differently in different countries, leading to very wide variation in its current application in pregnancy. In this short review, we will discuss the history of metformin in pregnancy and highlight some of the key clinical trials. We will then consider some of the remaining controversies associated with metformin use in pregnancy, most important of these being the potential for long-term 'programming' effects on the fetus as a result of metformin being able to cross the placenta. We will also consider clinical situations where metformin might be avoided. Finally, we will discuss some future directions for this drug as it reaches its sixtieth anniversary.
\end{abstract}

Keywords Metformin $\cdot$ Pregnancy $\cdot$ Review $\cdot$ Teratogenesis

Electronic supplementary material The online version of this article (doi:10.1007/s00125-017-4351-y) contains a slide of the figure for download, which is available to authorised users.

Robert S. Lindsay

robert.lindsay@glasgow.ac.uk

1 Institute of Cardiovascular and Medical Sciences, British Heart Foundation (BHF) Glasgow Cardiovascular Research Centre, University of Glasgow, 126 University Place, Glasgow G12 8TA, UK

2 Section on Islet Cell and Regenerative Biology, Joslin Diabetes Center, Boston, MA, USA

\author{
Abbreviations \\ AMPK AMP-activated protein kinase \\ ESC Embryonic stem cells \\ GDM Gestational diabetes \\ MATE Multidrug toxin and extrusion \\ MiG Metformin in Gestational Diabetes \\ mTOR Mechanistic target of rapamycin \\ OCT Organic cation transporter \\ PCOS Polycystic ovarian syndrome
}

\section{Early use}

The initial development and use of metformin (outside of pregnancy) are reviewed elsewhere in this issue of Diabetologia [1]. With regard to pregnancy, it is important to note that it was acknowledged very early on that metformin crossed the placenta. More recent studies show similar plasma concentrations in the maternal and fetal circulation [2]. Further, the combination of increased lactic acidosis risk (mainly observed with the metformin-related biguanide, phenformin) and the relatively hypoxic fetal environment led to important concerns regarding potential adverse effects of metformin use in pregnancy, for both mother and child. In fact, the safety concerns related to phenformin use resulted in the withdrawal of metformin in many, although not all, countries [3].

These early concerns are charted in influential reports of the Aberdeen International Colloquia on Carbohydrate Metabolism in Pregnancy and the Newborn. The first colloquium, reported in 1975, included an entire chapter on 'the use of sulphonylureas, biguanides and insulin in pregnancy' [4]. By the time of the fourth report in 1988, the topic of use of metformin was given only a few lines and it was noted that use was not widespread [5]. 
Metformin use did, however, continue in other parts of the world. In developing countries, the relatively low cost of metformin compared with insulin made it an attractive option. Coetzee and colleagues published a series of important observational papers, commencing in the late 1970s, examining the use of metformin in South Africa [6-8]. In South Africa and other countries, where metformin was routinely used to treat type 2 diabetes, exposure inevitably began to occur in early pregnancy leading to the separate analysis of safety in early pregnancy, particularly regarding miscarriage and congenital anomaly.

However, it was as metformin use became more popular in polycystic ovarian syndrome (PCOS) that a more robust literature developed, investigating exposure of the fetus to metformin in early pregnancy and, therefore, safety of its use.

\section{Safety and efficacy of metformin during pregnancy}

Safety in early pregnancy The early literature regarding metformin use in early pregnancy in humans was based on observational findings and of variable quality. Studies were usually small and it was often difficult to tease out the potential teratogenic effects of metformin, particularly as opposed to the well-established effects of maternal hyperglycaemia to increase risk of congenital malformation [9]. Similarly, animal studies have not been completely conclusive, and while increases in embryonic AMP-activated protein kinase (AMPK; AMPK activation being one potential effect of metformin) may be key in diabetic embryopathy, animal studies have not suggested an increase in embryopathy with early metformin exposure in vivo [10].

More recently, a meta-analysis based on metformin exposure in 351 women with PCOS has been carried out. Interestingly, the findings of this study do not suggest an increase in congenital anomaly with metformin use in pregnancy (OR of major birth defect 0.86 [95\% CI $0.18,4.08]$ ] [11]. However, this estimate is based on a small sample size and, therefore, the confidence intervals remain wide.

Metformin has also been extensively analysed in the context of PCOS-associated miscarriage and pregnancy induction. Its use appears to be neutral with regards to miscarriage rates, although some argue that it actually reduces rates of miscarriage [12], and it is superior when used either alone or in combination with clomifene for ovulation induction as compared with placebo [13].

Taken together, while there are important theoretical concerns, metformin appears to be safe in early pregnancy, with no convincing evidence for an increase in congenital malformations or miscarriage with its use. Indeed, in the most common clinical setting of metformin exposure in pregnancy, a woman with type 2 diabetes already on metformin in early pregnancy, the overriding clinical issue should be that discontinuation of use would potentially expose the patient to complications associated with poor glycaemic control. Early clinical assessment and careful consideration of tight control of blood glucose remain key.

Metformin use in gestational diabetes As previously mentioned, for some years the use of metformin was limited to specific geographical locations, such as Cape Town in South Africa, but the Metformin in Gestational Diabetes $(\mathrm{MiG})$ randomised clinical trial by Rowan et al in 2008 altered medical practice in many countries [14]. In this study, 751 women with gestational diabetes (GDM) were randomised to either metformin or usual treatment with insulin therapy [14]. The safety profile for mothers appeared to be good. Gastrointestinal side effects led to discontinuation of metformin in $1.9 \%$ of women and reduction in dose in $8.8 \%$. The primary outcome included a composite of neonatal hypoglycaemia $(<2.6 \mathrm{mmol} / \mathrm{l})$, respiratory distress, need for phototherapy, 5 min Apgar score $<7$ or premature birth (before 37 weeks), and was no different between the two treatment groups, being present in $32 \%$ of both the metformin- and insulin-treated participants. Secondary outcomes, including birthweight, neonatal anthropometrics and rates of large for gestational age ( $>90$ th percentile) were also equivalent between the groups. However, the rates of more-severe hypoglycaemia $(<1.6 \mathrm{mmol} / \mathrm{l})$ were reduced in the metformin group vs insulin therapy. It is important to note that some $46.3 \%$ of women in the metformin group required supplemental insulin treatment to maintain glycaemic control. Metformin appeared to have good patient acceptability, with $76.6 \%$ of women suggesting that they would choose metformin in a subsequent pregnancy compared with $27.2 \%$ of those initially assigned to insulin. Metformin was associated with less weight gain between enrolment in the trial and 36 or 37 weeks of pregnancy $(0.4 \pm 2.9 \mathrm{~kg}$ in the metformin group vs $2.0 \pm 3.3 \mathrm{~kg}$ in the insulin group; $p<0.001$ ) [14].

More recently, findings from a meta-analysis supported the safe use of metformin as a first-line treatment for GDM (after dietary interventions), showing that this drug has equivalent outcomes to primary insulin treatment with regards to its effects on newborns, and salutary effects on maternal weight gain [15]. Furthermore, this study suggested that metformin may have superior outcomes to the only other oral glucose-lowering agent used in pregnancy, glibenclamide (known as glyburide 
in the USA and Canada), although only few head-tohead studies were used in the analysis [15]. In support of these findings, a recent small RCT reported a similar safety and efficacy profile of metformin vs glibenclamide [16]. These outcomes are encouraging as, in general, metformin use is less expensive and generally easier for patients to administer than insulin. However, another detailed meta-analysis has also highlighted the limited extent of the evidence base for proper comparison of treatments [17].

Can we select which women are best suited for metformin therapy? Women who required supplemental insulin had a higher BMI in early pregnancy than those maintained on metformin $\left(33.6 \pm 8.6 \mathrm{~kg} / \mathrm{m}^{2}\right.$ vs $31.1 \pm 7.8 \mathrm{~kg} / \mathrm{m}^{2}$, insulin vs metformin), and higher baseline glucose levels $(6.1 \pm 1.1 \mathrm{mmol} / \mathrm{l})$ compared with those not requiring supplemental insulin $(5.3 \pm 0.8 \mathrm{mmol} / \mathrm{l})$ [14]. This is broadly similar for other glucose-lowering agents, such as glibenclamide, the failure of which is more likely when initial fasting glucose is high ( $>6.4 \mathrm{mmol} / \mathrm{l}$ ) [18], and these observations make sense, a priori, as women with higher glucose levels are likely to have more severe disease. Similarly, women presenting with GDM earlier in pregnancy are more likely to require insulin and may be considered less suitable for oral agents.

Metformin use in type $\mathbf{2}$ diabetes The familiarity of metformin in general diabetes practice and the recent encouraging results from the MiG trial have led to speculation as to its possible uses in women with type 2 diabetes in pregnancy. It is important to note, however, that the evidence base is very small.

Given the insulin resistance of pregnancy, it is likely that most women with type 2 diabetes before pregnancy will require treatment with insulin during their pregnancy, simply to maintain glycaemic control. Is there a role then for metformin as an insulin-sparing agent? There are only a few observational studies on the use of metformin in type 2 diabetes in pregnancy; in 2000, Hellmuth et al reported use of metformin in 50 women in Denmark, 19 of whom had type 2 diabetes [19]. In this retrospective study examining the historical practice with oral glucose-lowering agents between 1966 and 1984, an increase in pre-eclampsia and perinatal mortality was noted with metformin use compared with treatment with sulfonylureas or insulin [19]. Levitt and colleagues reported observational data from South Africa; in their extensive study, women with pregestational type 2 diabetes were treated with insulin or oral glucoselowering agents before and during pregnancy. Notably, this was also not a randomised study but reflected local practice. Critically, they observed a very high rate of perinatal mortality (125 events per 1000 births) in the group treated with oral glucose-lowering medication (predominantly metformin and glibenclamide) throughout pregnancy compared with women who converted from oral glucose-lowering agents to insulin (28 events per 1000 births) or women who converted from diet to insulin or remained on insulin during pregnancy (33 events per 1000 births) [8]. In contrast, in 2006 Hughes and Rowan reported observational data on the use of metformin in type 2 diabetes in the New Zealand population; they reported no increase in adverse pregnancy outcomes despite a worse risk-factor profile at baseline compared with women not taking metformin [20].

As detailed above, the evidence base for metformin use in pregestational type 2 diabetes is not strong, as trials were not randomised and liable to selection bias. Nevertheless, it would appear inadvisable to consider metformin as a sole agent for the management of women with type 2 diabetes in pregnancy. As a separate issue, there is a potential for use of metformin as an insulin-sparing agent with potential benefits on weight gain in pregnancy and, more, speculatively on glycaemic control. However, there is clearly not enough evidence at this time to recommend such use, with an obvious need for randomised evidence to elucidate the benefits, if any, of metformin as an additional agent for the management of type 2 diabetes in pregnancy. Currently ongoing randomised trials in this area will inform the debate in the future [21].

\section{Does metformin have long-term effects on the child after treatment in utero?}

As detailed above, there is not strong evidence of safety problems for metformin during pregnancy. Therefore, most of the lingering safety concerns revolve around potential long-term effects of metformin exposure in utero. At the time of writing, randomised evidence arising from metformin exposure during pregnancy, to treat GDM, extends to only 2 years, although longer-term studies are expected. In MiG: the offspring follow-up (MiG TOFU), children exposed to metformin in utero were shown to have no difference in total fat mass and body fat percentage, as assessed by bioimpedance, although children did have slightly larger mid-upper arm circumferences, subscapular and bicep skinfolds [22]. There were no differences in blood pressure [23]. Follow-up of a Finnish RCT found that children exposed to metformin in pregnancy were significantly heavier at the age of 12 months, and taller and heavier $(12.0 \mathrm{vs} 11.3 \mathrm{~kg})$ at 18 months, although the study was small, with a total of 97 children. The mean ponderal index (PI) did not differ significantly and there were no 
differences in motor, social and linguistic development evaluated at the age of 18 months [24]. Follow-up of a small RCT in women with PCOS suggested no difference in BMI at 8 years, but only included 25 children [25]. The limitations of size of study and length of follow-up in the available studies are noted in a recent review [26].

\section{Does recent basic science explain how use of metformin in pregnancy can affect the embryo or fetus?}

Is there malformation risk to the embryo? As noted above, increased embryo AMPK activity mediates some of the adverse effects of maternal diabetes on congenital malformations in mouse embryos, raising the concern that metformin stimulation of embryo AMPK activity could counteract the beneficial effects of lowering maternal blood glucose levels [27]. In addition, the effects of metformin on one-carbon pathways, which are similar to the effects of anti-folate chemotherapeutic drugs [28], and the essential role of folate in preventing neural tube (and other) malformations [29] raises further concern as to whether metformin might increase embryo malformation risk. However, at doses that stimulated maternal liver AMPK activity, metformin did not stimulate embryo AMPK activity or increase congenital anomalies [10]. Metformin did, however, stimulate AMPK activity and inhibit expression of a gene associated with congenital malformations in mouse embryonic stem cells (ESC) that were used as an in vitro model to study diabetic embryopathy [10]. Interestingly, the difference in metformin susceptibility between mouse embryos and mouse ESC was related to differential expression of metformin transporters [10]. Metformin is transported by organic cation transporters (OCTs) the normal function of which is to take up and extrude organic cations, such as thiamine, choline, neurotransmitters, creatinine, carnitine, guanidine and steroid hormones [30]. Slc47al and Slc22a3 mRNA, encoding the metformin transporters multidrug toxin and extrusion (MATE) 1 and OCT3, respectively, were expressed at negligible or undetectable levels in embryos, but Slc22a3 was expressed by ESC, albeit at much lower levels than by maternal liver [10]. The differences in metformin transporter expression and metformin responsiveness between normal mouse embryos and ESC could be a result of metabolic differences between ESC lines in culture and the normal embryo in vivo. It should be noted that the ESC line that was used to study potential teratogenic effects of metformin [10] does not exhibit the responsiveness to high glucose exposure and the high rates of glycolysis relative to oxidative phosphorylation of normal embryos or of ESC derived in physiological glucose media [31]. While conventional stem cell (embryonic and induced pluripotent) lines exhibit fewer, and less mature, mitochondria compared with more differentiated cells, and high rates of glycolysis relative to oxidative phosphorylation are essential for induction of pluripotency from differentiated cells [32-34], conventional ESCs may be more dependent on mitochondrial metabolism (and less dependent on glycolysis) than the normal mouse embryo in vivo. One can speculate that the endogenous solutes taken up by metformin transporters (OCTs) have a function in mitochondrial metabolism, such that metformin transporters would not be expressed by cells that are highly dependent on glycolysis, but would occur upon maturation of mitochondrial respiration (Fig. 1). Consistent with this, metformin and phenformin competitively inhibit thiamine uptake by hepatocytes, causing inhibition of the tricarboxylic acid cycle [35]. Whether the human embryo also lacks metformin transporter expression and metformin responsiveness is not known. However, preimplantation human embryos have low mitochondrial content and are dependent on anaerobic metabolism [32], suggesting that early human embryos may be unresponsive to metformin.

Are there metabolic programming effects on the fetus? Compared to the embryo, fetal and placental cells are more differentiated and more dependent on oxidative metabolism and mitochondrial activity. Metformin inhibits complex I of the respiratory chain, leading to an increased AMP:ATP ratio that stimulates AMPK activity [36-38]. AMPK regulates several processes including gene expression and mechanistic target of rapamycin (mTOR)-induced effects on protein synthesis [27, 39-41]. In addition, there are separated, but nonredundant mitochondrial and cytosolic pathways that generate onecarbon intermediates that can be inhibited by metformin, mimicking the 'methyl folate trap' that can occur during vitamin $\mathrm{B}_{12}$ deficiency $[28,42,43]$. This can lead to methionine deprivation, decreased glutathione (reduced, oxidised disulphide and trisulphide forms), increased homocysteine, and decreased de novo synthesis of purines and pyrimidines [28, 42]. In addition, inhibition of onecarbon metabolism decreases levels of Sadenosylmethionine (SAM) and increases levels of Sadenosylhomocysteine (SAH), which could have epigenetic effects on gene expression because of reduced DNA and histone methylation. Further, a recent study using the round worm Caenorhabditis elegans showed that metformin inhibits cell growth and promotes longevity through inhibition of mitochondrial respiration, leading to reduced transit of the Ras-related GTP-binding protein $\mathrm{C}$ ( $\mathrm{RagC}$ ) 


\section{a}

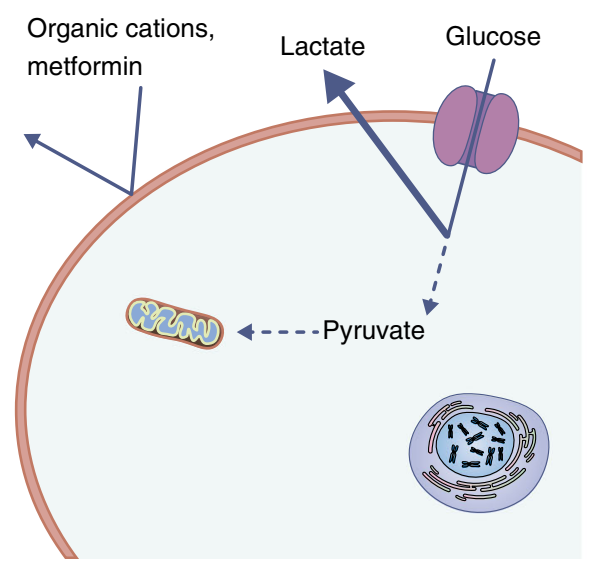

Fig. 1 Model showing that susceptibility to metformin depends on the presence of metformin transporters and maturity of mitochondrial function. (a) Early post-implantation embryo cells (and, possibly, fetal progenitor cells) are metformin-resistant because of negligible transporter expression. Metabolism is predominantly glycolytic and mitochondrial content is low. (b) Differentiated placental ESC (conditioned for high

GTPase through the nuclear pore complex (NPC) and inhibition of mTOR signalling [44]. This pathway is highly conserved throughout evolution and may explain the anti-cancer effect of metformin, because the same metformin response reduces viability of melanoma and pancreatic cancer cells [44]. These observations raise the question: since metformin appears to use some of the same mechanisms as nutrient restriction to promote longevity and inhibit cancer growth [42,45], and dietary restriction (and vitamin $\mathrm{B}_{12}$ deprivation) can programme increased cardiometabolic risk in the offspring [46, 47], could metformin exert long-term negative effects on cardiometabolic risk in the offspring?

In order for metformin to affect fetal or placental physiology and development, the cells in these tissues must be able to take up metformin. Furthermore, because metformin is positively charged at neutral $\mathrm{pH}$, there must be a strong mitochondrial membrane potential for it to enter the mitochondrial matrix [48]. Human placentas express several OCT isoforms (OCT1, OCT2, OCT3, and MATE1 and MATE2) [49], and metformin may indirectly affect fetal development, for example, through altered nutrient delivery or placental growth; although, this requires further investigation. Whether fetal tissues express OCTs has not been carefully studied. It should be noted that metformin may not have lasting effects on organs whose growth and repopulation depends on progenitor cells, unless those progenitor cells take up and are metabolically or epigenetically altered by metformin. Yet, in the case of the liver, where it appears that hepatocytes are derived primarily from pre-existing hepatocytes rather than stem cells [50, 51], the epigenetic effects of metformin could have long-term outcomes if chromatin modifications are passed onto daughter cells. b

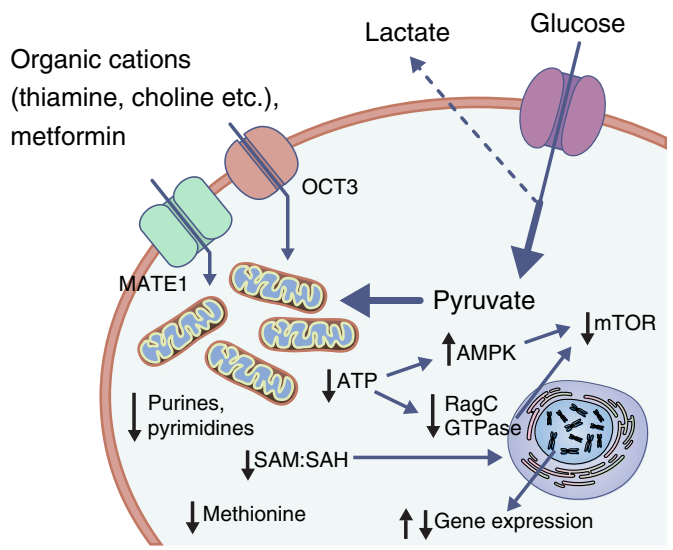

glucose culture). Caenorhabditis elegans cells (and, potentially, fetal cells) express higher levels of metformin transporters and are metformin-responsive through effects on mitochondria and gene expression. Metabolism is predominantly oxidative and mitochondrial content is high. RagC, Ras-related GTP-binging protein C; SAH, Sadenosylhomocysteine; SAM, S-adenosylmethionine

\section{International use of metformin}

Metformin use appears quite varied between countries. In New Zealand, guidelines published by the Ministry of Health in 2014, after extensive review of the evidence, recommended 'where women who have gestational diabetes and

\section{Metformin use in pregnancy: summary of evidence}

The evidence outlined below is majorly based on small sample sizes and non-randomised trial data and must, therefore, be interpreted with caution.

GDM Good safety profile in mothers; reduced rates of severe hypoglycaemia $(<1.6 \mathrm{mmol} / \mathrm{l})$ vs other agents; reduced pregnancy weight gain vs insulin; superior to glibenclamide for glucose control; more likely to fail in women with higher baseline glucose or presenting with diabetes earlier in pregnancy. Similar effects on newborn health vs insulin.

Type 2 diabetes Only observational evidence.

Long-term effects in offspring Equivocal evidence for changes in body composition; no differences in BP; no differences in motor, social and linguistic development at 18 months.

Fetal effects No convincing evidence for increase in congenital malformations or miscarriage; longterm effects on health or metabolism during adulthood not known. 
poor glycaemic control (above treatment targets) in spite of dietary and lifestyle interventions, offer oral hypoglycaemics (metformin or glibenclamide) and/or insulin therapy' [52]. In Scotland, UK, the Scottish Intercollegiate Guidelines Network (SIGN publication no. 116; 2010) suggested that 'metformin or glibenclamide may be considered as initial pharmacologi$\mathrm{cal}$, glucose-lowering treatment in women with gestational diabetes' [53].

In contrast, the most recent ADA standard of medical care notes that insulin is the first-line agent recommended for treatment of GDM in the USA [54]. Metformin was noted to have randomised evidence for safety and efficacy, but the lack of long-term safety data for offspring was also noted. The US Food and Drug Administration (FDA) places metformin in category B: 'Animal reproduction studies have failed to demonstrate a risk to the fetus, and there are no adequate and wellcontrolled studies in pregnant women' [54].

In England and Wales (UK), the National Institute for Health and Care Excellence (NICE) recommends that clinicians 'offer metformin to women with gestational diabetes if blood glucose targets are not met using changes in diet and exercise within 1-2 weeks'. However, at the same time, these recommendations note that the summary of product characteristics for metformin suggests that it should not be used in pregnancy but, instead, insulin should be used [55].

Australian guidelines issued by the Royal Australian College of General Practitioners note that 'metformin has been used internationally as initial glucose-lowering treatment in women with GDM. However, it has not been approved for this use in Australia for this indication. Lifestyle and insulin therapy remain the mainstay of therapy' [56].

\section{Conclusions}

International use of metformin in pregnancy has increased greatly in the last decade. The drug is familiar, relatively inexpensive and easy to administer, and is associated with clear benefits as a treatment of hyperglycaemia in pregnancy. For many clinicians, a major concern is the stopping of metformin early in pregestational type 2 diabetes, without adequate replacement, potentially worsening hyperglycaemia. At the same time, as a community, clinicians treating women with diabetes in pregnancy are appropriately, very sensitive to the potential for long-term adverse outcomes. At the time of writing, there certainly do not appear to be clear data suggesting a long-term problem with metformin use, but some clinicians will want to await further data. One troubling aspect of this is that, given the relatively small size of funded studies, difficulty of maintaining cohorts in the longer term, and the potential for high attrition rates, we may never have absolutely clear evidence for this. On its sixtieth anniversary then, perhaps, metformin stands as an exemplar of how research in the management of medical conditions in pregnancy must develop. Those on either side of the debate about its use will agree that better data should have been available earlier and hope that the debate will be able to move on prior to metformin's next significant anniversary.

Funding RSL has received speaker fees and advisory board fees from Eli Lilly Ltd and Novo Nordisk Ltd. MRL has received funding from the National Institute of Health (NIH R01DK104649 to MRL and NIH P30DK036836 to the Joslin Diabetes Center). No specific grant from any funding agency in the public, commercial or not-for-profit sectors was received for this article.

Duality of interest The authors declare that there is no duality of interest associated with this manuscript.

Contribution statement RSL and MRL were both involved in the conception, design, drafting of the article, and final approval of the version to be published.

Open Access This article is distributed under the terms of the Creative Commons Attribution 4.0 International License (http:// creativecommons.org/licenses/by/4.0/), which permits unrestricted use, distribution, and reproduction in any medium, provided you give appropriate credit to the original author(s) and the source, provide a link to the Creative Commons license, and indicate if changes were made.

\section{References}

1. Bailey CJ (2017) Metformin: historical overview. Diabetologia doi: 10.1007/s00125-017-4318-Z

2. Charles B, Norris R, Xiao X, Hague W (2006) Population pharmacokinetics of metformin in late pregnancy. Ther Drug Monit 28:6772

3. Bailey CJ, Turner RC (1996) Metformin. N Engl J Med 334:574579

4. Sutherland HWSJ (1975) The use of sulphonylureas biguanides and insulin in pregnancy. In: Sutherland HWSJ (ed) Carbohydrate metabolism in pregnancy and the newborn. Churchill Livingstone, Edinburgh

5. Maresh MJBR (1988) Screening and management of gestational diabetes mellitus. In: Sutherland HWSJ, Pearson DW (eds) Carbohydrate metabolism in pregnancy and the newborn, 4th edn. Springer Verlag, London

6. Coetzee EJ (2007) Counterpoint: oral hypoglyemic agents should be used to treat diabetic pregnant women. Diabetes Care 30:2980 2982

7. Coetzee EJ, Jackson WP (1979) Metformin in management of pregnant insulin-independent diabetics. Diabetologia 16:241-245

8. Ekpebegh CO, Coetzee EJ, van der Merwe L, Levitt NS (2007) A 10 -year retrospective analysis of pregnancy outcome in pregestational type 2 diabetes: comparison of insulin and oral glucose-lowering agents. Diabet Med 24:253-258

9. Piacquadio K, Hollingsworth DR, Murphy H (1991) Effects of inutero exposure to oral hypoglycaemic drugs. Lancet 338:866-869

10. Lee HY, Wei D, Loeken MR (2014) Lack of metformin effect on mouse embryo AMPK activity: implications for metformin treatment during pregnancy. Diabetes Metab Res Rev 30:23-30

11. Cassina M, Dona M, Di Gianantonio E, Litta P, Clementi M (2014) First-trimester exposure to metformin and risk of birth defects: a 
systematic review and meta-analysis. Hum Reprod Update 20:656669

12. Feng L, Lin XF, Wan ZH, Hu D, Du YK (2015) Efficacy of metformin on pregnancy complications in women with polycystic ovary syndrome: a meta-analysis. Gynecol Endocrinol 31:833-839

13. Wang R, Kim BV, van Wely M et al (2017) Treatment strategies for women with WHO group II anovulation: systematic review and network meta-analysis. BMJ 356:j138

14. Rowan JA, Hague WM, Gao W, Battin MR, Moore MP (2008) Metformin versus insulin for the treatment of gestational diabetes. N Engl J Med 358:2003-2015

15. Balsells M, Garcia-Patterson A, Sola I, Roque M, Gich I, Corcoy R (2015) Glibenclamide, metformin, and insulin for the treatment of gestational diabetes: a systematic review and meta-analysis. BMJ 350:h102

16. Nachum Z, Zafran N, Salim R et al (2017) Glyburide versus metformin and their combination for the treatment of gestational diabetes mellitus: a randomized controlled study. Diabetes Care 40: 332-337

17. Brown J, Martis R, Hughes B, Rowan J, Crowther CA (2017) Oral anti-diabetic pharmacological therapies for the treatment of women with gestational diabetes. Cochrane Database Syst Rev, Issue 1, Art. no.: CD011967. DOI: 10.1002/14651858.CD011967.pub2

18. Moore TR (2007) Glyburide for the treatment of gestational diabetes. A critical appraisal. Diabetes Care 30(Suppl 2):S209-S213

19. Hellmuth E, Damm P, Molsted-Pedersen L (2000) Oral hypoglycaemic agents in 118 diabetic pregnancies. Diabet Med 17:507-511

20. Hughes RC, Rowan JA (2006) Pregnancy in women with type 2 diabetes: who takes metformin and what is the outcome? Diabet Med 23:318-322

21. Feig DS, Murphy K, Asztalos E et al (2016) Metformin in women with type 2 diabetes in pregnancy (MiTy): a multi-center randomized controlled trial. BMC Pregnancy Childbirth 16:173

22. Rowan JA, Rush EC, Obolonkin V, Battin M, Wouldes T, Hague WM (2011) Metformin in gestational diabetes: the offspring followup (MiG TOFU): body composition at 2 years of age. Diabetes Care 34:2279-2284

23. Battin MR, Obolonkin V, Rush E, Hague W, Coat S, Rowan J (2015) Blood pressure measurement at two years in offspring of women randomized to a trial of metformin for GDM: follow up data from the MiG trial. BMC Pediatr 15:54

24. Ijas H, Vaarasmaki M, Saarela T, Keravuo R, Raudaskoski T (2015) A follow-up of a randomised study of metformin and insulin in gestational diabetes mellitus: growth and development of the children at the age of 18 months. BJOG 122:994-1000

25. Ro TB, Ludvigsen HV, Carlsen SM, Vanky E (2012) Growth, body composition and metabolic profile of 8-year-old children exposed to metformin in utero. Scand J Clin Lab Invest 72:570-575

26. Butalia S, Gutierrez L, Lodha A, Aitken E, Zakariasen A, Donovan L (2017) Short- and long-term outcomes of metformin compared with insulin alone in pregnancy: a systematic review and metaanalysis. Diabet Med 34:27-36

27. Wu Y, Viana M, Thirumangalathu S, Loeken MR (2012) AMPactivated protein kinase mediates effects of oxidative stress on embryo gene expression in a mouse model of diabetic embryopathy. Diabetologia 55:245-254

28. Corominas-Faja B, Quirantes-Pine R, Oliveras-Ferraros C et al (2012) Metabolomic fingerprint reveals that metformin impairs one-carbon metabolism in a manner similar to the antifolate class of chemotherapy drugs. Aging 4:480-498

29. Blom HJ (2009) Folic acid, methylation and neural tube closure in humans. Birth Defects Res Part A Clin Mol Teratol 85:295-302

30. Liang Y, Li S, Chen L (2015) The physiological role of drug transporters. Protein Cell 6:334-350
31. Jung JH, Wang XD, Loeken MR (2013) Mouse embryonic stem cells established in physiological-glucose media express the high KM Glut2 glucose transporter expressed by normal embryos. Stem Cells Transl Med 2:929-934

32. Cho YM, Kwon S, Pak YK et al (2006) Dynamic changes in mitochondrial biogenesis and antioxidant enzymes during the spontaneous differentiation of human embryonic stem cells. Biochem Biophys Res Commun 348:1472-1478

33. Xu X, Duan S, Yi F, Ocampo A, Liu GH, Izpisua Belmonte JC (2013) Mitochondrial regulation in pluripotent stem cells. Cell Metab 18:325-332

34. Zhou Y, Al-Saaidi RA, Fernandez-Guerra P et al (2017) Mitochondrial spare respiratory capacity is negatively correlated with nuclear reprogramming efficiency. Stem Cells Dev 26:166176

35. Chen L, Shu Y, Liang X et al (2014) OCT1 is a high-capacity thiamine transporter that regulates hepatic steatosis and is a target of metformin. Proc Natl Acad Sci U S A 111:9983-9988

36. El-Mir MY, Nogueira V, Fontaine E, Averet N, Rigoulet M, Leverve X (2000) Dimethylbiguanide inhibits cell respiration via an indirect effect targeted on the respiratory chain complex I. J Biol Chem 275:223-228

37. Owen MR, Doran E, Halestrap AP (2000) Evidence that metformin exerts its anti-diabetic effects through inhibition of complex 1 of the mitochondrial respiratory chain. Biochem J 348:607-614

38. Bridges HR, Jones AJ, Pollak MN, Hirst J (2014) Effects of metformin and other biguanides on oxidative phosphorylation in mitochondria. Biochem J 462:475-487

39. Zhou G, Myers R, Li Y et al (2001) Role of AMP-activated protein kinase in mechanism of metformin action. J Clin Invest 108:11671174

40. Lee M, Hwang JT, Lee HJ et al (2003) AMP-activated protein kinase activity is critical for hypoxia-inducible factor-1 transcriptional activity and its target gene expression under hypoxic conditions in DU145 cells. J Biol Chem 278:39653-39661

41. Weinberg SE, Chandel NS (2015) Targeting mitochondria metabolism for cancer therapy. Nat Chem Biol 11:9-15

42. Cabreiro F, Au C, Leung KY et al (2013) Metformin retards aging in $C$. elegans by altering microbial folate and methionine metabolism. Cell 153:228-239

43. Luciano-Mateo F, Hernandez-Aguilera A, Cabre N et al (2017) Nutrients in energy and one-carbon metabolism: learning from metformin users. Nutrients 9:121

44. Wu L, Zhou B, Oshiro-Rapley N et al (2016) An ancient, unified mechanism for metformin growth inhibition in C. elegans and cancer. Cell 167:1705-1718

45. Mair W, Dillin A (2008) Aging and survival: the genetics of life span extension by dietary restriction. Annu Rev Biochem 77:727754

46. Hales CN, Barker DJ (1992) Type 2 (non-insulin-dependent) diabetes mellitus: the thrifty phenotype hypothesis. Diabetologia 35 : 595-601

47. Yajnik CS, Deshpande SS, Jackson AA et al (2008) Vitamin B12 and folate concentrations during pregnancy and insulin resistance in the offspring: the Pune Maternal Nutrition Study. Diabetologia 51: 29-38

48. Wheaton WW, Weinberg SE, Hamanaka RB et al (2014) Metformin inhibits mitochondrial complex I of cancer cells to reduce tumorigenesis. Elife $3: \mathrm{e} 02242$

49. Ahmadimoghaddam D, Zemankova L, Nachtigal P et al (2013) Organic cation transporter 3 (OCT3/SLC22A3) and multidrug and toxin extrusion 1 (MATE1/SLC47A1) transporter in the placenta and fetal tissues: expression profile and fetus protective role at different stages of gestation. Biol Reprod 88:55

50. Duncan AW, Dorrell C, Grompe M (2009) Stem cells and liver regeneration. Gastroenterology 137:466-481 
51. Malato Y, Naqvi S, Schurmann N et al (2011) Fate tracing of mature hepatocytes in mouse liver homeostasis and regeneration. J Clin Invest 121:4850-4860

52. Ministry of Health - Manatū Hanoura (2014) Screening, diagnosis and management of gestational diabetes in New Zealand: a clinical practice guideline. Available from https://www.health.govt.nz/ system/files/documents/publications/screening-diagnosismanagement-of-gestational-diabetes-in-nz-clinical-practiveguideline-dec14-v2.pdf. Accessed 5 May 2017

53. Scottish Intercollegiate Guidelines Network (2014) Management of diabetes: a national clinical guideline (SIGN publication no. 116.) Available from www.sign.ac.uk/pdf/sign116.pdf. Accessed 5 May 2017
54. American Diabetes Association (2017) Management of diabetes in pregnancy. Diabetes Care 40(Suppl 1):S114-S119

55. National Institute for Health and Care Excellence (2015) Diabetes in pregnancy: management of diabetes and its complications from preconception to the postnatal period. Available from www.nice. org.uk/guidance/ng3. Accessed 5 May 2017

56. The Royal Australian College of General Practitioners (2016) Gestational diabetes mellitus. In: General practice management of type 2 diabetes: 2016-18. Available from www.racgp.org.au/yourpractice/guidelines/diabetes/13-diabetes-and-reproductive-health/ 133-gestational-diabetes-mellitus/. Accessed 5 May 2017 